\title{
A Solution to the Problem of X-Ray Mapping Samples with Surface Roughness
}

\author{
R. Anderhalt, D. Chan, and M. Lupu
}

EDAX Inc., 91 McKee Drive, Mahwah, NJ, 07430

X-ray mapping by EDS techniques of rough samples or samples with a complex topographic surface is more difficult than mapping relatively smooth surfaces. The problem of the sample with a rough surface arises because a lower intensity of an element, or even no intensity, can either mean the absence of that element or it can mean the element has a significant presence but the area can not be "viewed" by the EDS detector (Fig. 1). This problem or ambiguity can be partially solved by the use of a second detector which would ideally have an opposing view. The use of a second detector still presents some problems of interpretation in that there is a contrast artifact arising from the fact that some areas of the sample produce $\mathrm{x}$ rays which can be received by both detectors, while the $\mathrm{x}$ rays from other areas are detected by only one or by neither of the two detectors [1]. This artifact originates from the simple summing of the $\mathrm{x}$ rays from the two detectors (Fig. 1c). A method is presented that can work around this problem and will provide a better interpretation of the chemical variability within the sample.

When two detectors are used each detector will have its own spectral map dataset or data cube and the two datasets are merged in a variety of ways to produce a third dataset. The merging can be done by the summing or averaging of the spectral data at each pixel (Fig. 1c). A preferred method would be to merge only the spectral information from the detector that had the most counts at each pixel and to ignore the counts from the detector that had a lower, or a much lower intensity (Fig. 1d). Although it might seem counter-intuitive that some data should be discarded, this eliminates most of the problem of the contrast artifact and will tend to equalize the contrast between areas that are able to have $\mathrm{x}$ rays counted by both detectors as compared to those areas where the $\mathrm{x}$ rays are seen by only one detector. The contrast artifact is often greatly diminished but not completely eliminated by selecting the information from the detector with the maximum intensity. The chemical contrast may be enhanced as compared to topographic effects by an additional normalization step. The normalization procedure that has been used to a positive effect is a full quantification (ZAF Wt. \%) of the merged map data (Fig. 2).

There is still a need to either eliminate the data for the pixels that are shadowed in the view of both detectors or to indicate in some way that the data in the maps for these regions are not reliable (note the noisy data in Fig. 2a from some of the voids). A map can be created from the summing of all counts of all energies on a pixel by pixel basis for each detector and these maps can be summed for the 2 detector case. Dark areas of this map represent areas in the shadow of both detectors. If the map is inverted and rendered as a binary image, it can be used as an overlay against a single map or with several maps (Figs. 1 and 2) and the "null" areas where no x rays are detected will be shown in white. When overlain with a map of a single element, there will be a clear indication of areas that do not contain the element as compared to the areas with a problematic topography.

References

[1] R. Anderhalt et al., Microsc. Microanal. 14 (Suppl 2) (2008) 1102. 

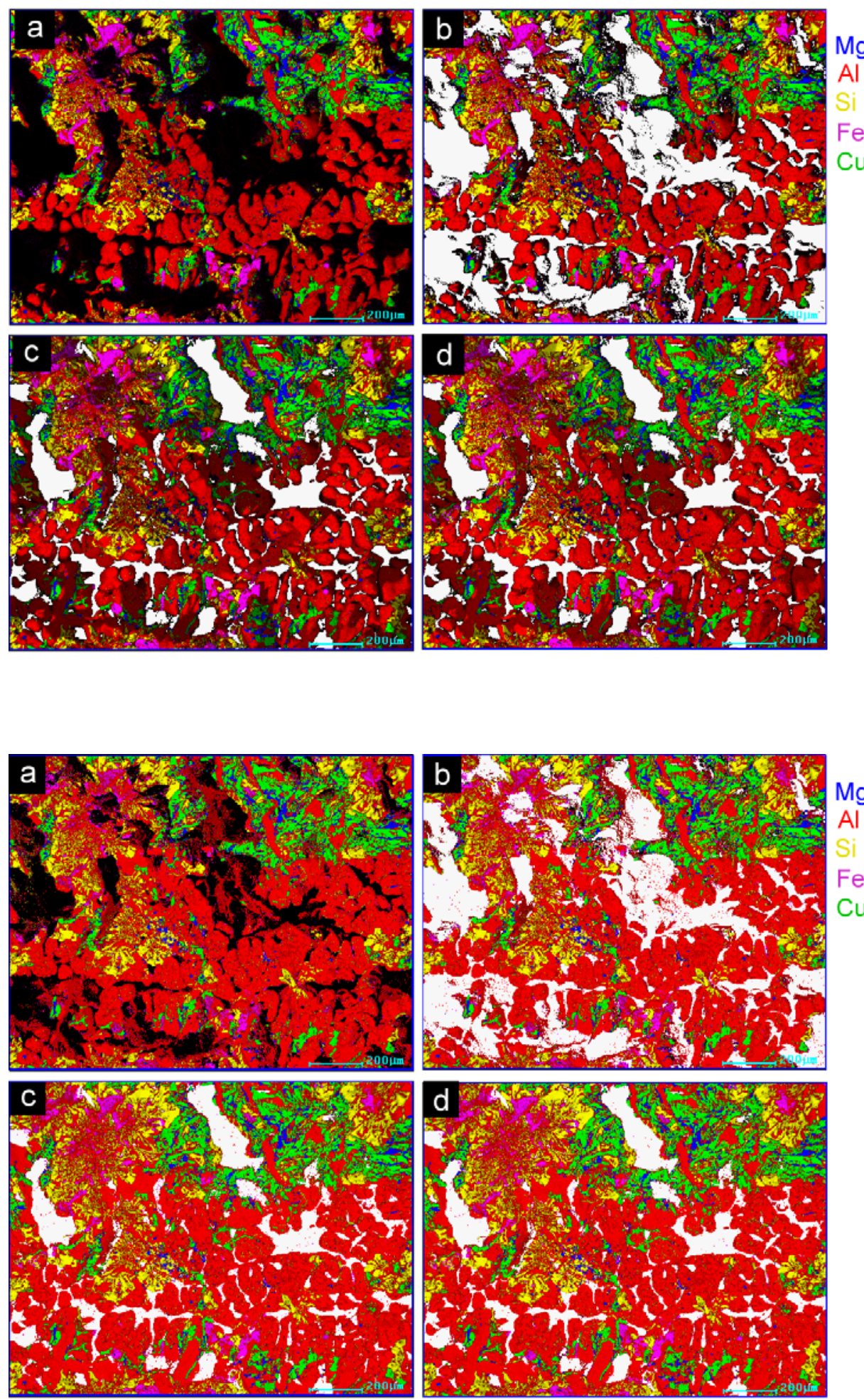

Figure 1. Map overlay of the x-ray intensity of $\mathrm{Mg}$ (blue), $\mathrm{Al}$ (red), Si (yellow), Fe (magenta) and $\mathrm{Cu}$ (green) from a fracture surface of an aluminum alloy (beam energy $15 \mathrm{keV}$, field width is 1.5 $\mathrm{mm}$ ). The images in a) and b) are from a single detector. The images in c) and d) have resulted from the usage of 2 EDS detectors. In a) the dark area may represent an area with no x-ray intensity or a missing element. In b), c) and d) the areas having very few detected $\mathrm{x}$ rays are shown in white. In Fig. c) the data from each of two detectors was summed. In d) the maximum intensity at each pixel was selected.

$\mathrm{Mg}$ Figure 2. Map overlays of the Al same sample as in Fig. 1. Instead of mapping x-ray intensity, the spectral information at each pixel has been quantified (ZAF Wt. \%) in an attempt to normalize some of the variability observed in Fig. 1. In a) the dark area may represent an area with minimal x-ray intensity that gives a poor quantification and noisy data. In b), c) and d) the areas having no detected $x$ rays are shown in white. In b) the noisy data is effectively removed. In Fig. c) the data from each of two detectors was summed and then quantified. In d) the maximum intensity at each pixel was selected and then quantified. 